\title{
Feasibility and efficacy of transthoracic single-port assisted laparoscopic esophagogastrectomy for Siewert type II adenocarcinoma of the esophagogastric junction
}

\author{
Sijing Luo ${ }^{1 \#}$, Jiamin $\mathrm{Xu}^{2 \#}$, Wenjun Xiong ${ }^{3 \#}$, Jin $\mathrm{Li}^{3}$, Lijie Luo ${ }^{3}$, Yansheng Zheng ${ }^{3}$, Haiping Zeng ${ }^{2}$, \\ Yangwen $\mathrm{Liu}^{4}$, Licong Yang ${ }^{4}$, Zhengqian $\mathrm{Wu}^{4}$, Xiaobo Yang ${ }^{5}$, Wei Wang ${ }^{2,3}$ \\ ${ }^{1}$ General Surgery 1, Guangdong Provincial Hospital of Chinese Medicine, Zhuhai, China; ${ }^{2}$ The Second Clinical College of Guangzhou University \\ of Chinese Medicine, Guangzhou, China; ${ }^{3}$ Gastrointestinal Surgery, Guangdong Provincial Hospital of Chinese Medicine, Guangzhou, China; \\ ${ }^{4}$ General Surgery 1, Zhaotong Hospital of Chinese Medicine, Zhaotong, China; ${ }^{5}$ State Key Laboratory of Dampness Syndrome of Chinese Medicine, \\ Guangdong Provincial Hospital of Chinese Medicine, Guangzhou, China \\ Contributions: (I) Conception and design: W Wang, X Yang; (II) Administrative support: W Wang; (III) Provision of study materials or patients: W \\ Wang, W Xiong, L Luo, Y Zheng, J Li; (IV) Collection and assembly of data: S Luo, J Xu; (V) Data analysis and interpretation: S Luo, J Xu, W \\ Xiong; (VI) Manuscript writing: All authors; (VII) Final approval of manuscript: All authors. \\ \#These authors contributed equally to this work. \\ Correspondence to: Wei Wang. Department of Gastrointestinal Surgery, Guangdong Provincial Hospital of Chinese Medicine, Dade Road No. 111, \\ 510120, Guangzhou, China. Email: wangwei16400@163.com; Xiaobo Yang. Guangdong Provincial Hospital of Chinese Medicine, Dade Road No. \\ 111, Guangzhou 510120, China. Email: yangxiaobomd@163.com.
}

Background: The surgical treatment of Siewert type II adenocarcinoma of the esophagogastric junction (AEG) is controversial, and no systematic technology has been established. The aim of this retrospective study is to introduce the technology of transthoracic single-port assisted laparoscopic esophagogastrectomy.

Methods: Data from patients with Siewert type II AEG who underwent transthoracic single-port assisted laparoscopic esophagogastrectomy in Guangdong Provincial Hospital of Chinese Medicine from May 2017 to December 2020 were analyzed.

Results: A total of 35 patients, including 30 males and 5 females, were enrolled in this study. Eight patients underwent proximal gastrectomy while the other 27 patients underwent total gastrectomy. The median operative times were 247.5 (195.0-275.0) $\mathrm{min}$ and 290.0 (173.0-530.0) min for proximal and total gastrectomy, respectively. The median lower mediastinal lymph node dissection (LMLD) time was 41.5 (20.0-57.0) min and the median estimated blood loss was 100.0 (20.0-200.0) $\mathrm{mL}$. The median number of harvested mediastinal lymph nodes was 5 [2-13]. Lower mediastinal lymph node metastasis occurred in 9 patients $(25.7 \%)$. The lower mediastinal lymph node metastasis rate was significantly higher in patients with esophageal involvement exceeding $2 \mathrm{~cm}[>2$ vs. $\leq 2 \mathrm{~cm}: 55.6 \%(5 / 9)$ vs. 15.4\% (4/26), $\mathrm{P}=0.03]$. The median postoperative hospital stay was 10 [6-73] days. Overall morbidity was $11.8 \%$ (4 patients), including 2 cases of pleural effusion, 1 case of pancreatic fistula, and 1 case of anastomotic leakage.

Conclusions: Transthoracic single-port assisted laparoscopic esophagogastrectomy is safe and feasible. It has the advantages of reducing the difficulty of LMLD and digestive tract reconstruction.

Keywords: Siewert classification; adenocarcinoma of the esophagogastric junction (AEG); lower mediastinal lymph nodes; lymphadenectomy

Submitted Aug 11, 2021. Accepted for publication Oct 02, 2021.

doi: $10.21037 / \mathrm{atm}-21-4574$

View this article at: https://dx.doi.org/10.21037/atm-21-4574 


\section{Introduction}

In recent years, with the incidence rate of adenocarcinoma of the esophagogastric junction (AEG) increasing, its treatment has gained more and more attention. Although chemotherapy and chemoradiotherapy play important roles, surgery is still the primary curative treatment for advanced AEG (1). However, the surgical approach for AEG is controversial. Traditional surgical approaches include transthoracic approach, transabdominal approach and thoracoabdominal approach. The Siewert classification has important guiding significance for the selection of AEG surgical methods, and is used worldwide (2). Among them, the surgical treatment of Siewert type II AEG is the most controversial.

According to the 5 th edition of the Japanese Gastric Cancer Treatment Guidelines, the resection extent of AEG include proximal gastrectomy + lower esophagectomy, total gastrectomy + lower esophagectomy, esophagectomy, total gastrectomy and upper gastrectomy. Lower mediastinal lymph node dissection (LMLD) is recommended for AEG which less than $40 \mathrm{~mm}$ in diameter, located at the esophagogastric junction (EGJ) or above (3). Based on the results of the JCOG9502 trial, the abdominal-transhiatal (TH) approach was recommended for Siewert type II and III AEG $(4,5)$. The CLASS-01 trial (6) and KLASS-02 trial (7) have provided strong evidence for laparoscopic distal gastrectomy for advanced gastric cancer. With the development of laparoscopic technology and the minimally invasive concept, laparoscopic techniques have been widely used for AEG (8-10). However, laparoscopic surgery for Siewert type II AEG is still technically difficult in terms of LMLD and digestive tract reconstruction. Herein, we propose a novel technique, transthoracic single-port assisted laparoscopic esophagogastrectomy, for advanced Siewert type II AEG. The purpose of this study is to explore the feasibility and efficacy of this technique. We present the following article in accordance with the STROBE reporting checklist (available at https://dx.doi.org/10.21037/atm-214574).

\section{Methods}

Between May 2017 and December 2020, 35 patients with advanced Siewert type II AEG who underwent transthoracic single-port assisted laparoscopic esophagogastrectomy at the Department of Gastrointestinal Surgery, Guangdong Provincial Hospital of Chinese Medicine were included in this study. The eligibility criteria included: (I) the tumor center was located between $1 \mathrm{~cm}$ above and $2 \mathrm{~cm}$ below the EGJ; (II) no upper and middle mediastinal lymph node enlargement was detected on the preoperative imaging examination; (III) no distant metastasis; (IV) the clinicopathological data are complete. The intraoperative (including operative time, blood loss, the number of harvested lymph gland and so on) and postoperative data (including postoperative complication, postoperative hospital stay and so on) were collected and analyzed to evaluate the safety and feasibility of this technique. All patients were given details about the operative procedure and potential risks before surgery, and provided written informed consent. All procedures performed in this study involving human participants were in accordance with the Declaration of Helsinki (as revised in 2013). The study was approved by the ethics committee of Guangdong Provincial Hospital of Chinese Medicine (ZF2018-219). It has been registered at ClinicalTrials.gov (NCT04423354).

\section{Special definition}

The time of LMLD was defined as the time from entry into the hiatus to transection of the esophagus.

\section{Surgical procedures}

\section{Surgical position and port arrangement}

After the induction of general anesthesia, patients were placed in a supine position with their legs apart and $20^{\circ}$ head-up tilt. Pneumoperitoneum was established after the insertion of a $10 \mathrm{~mm}$ trocar at the subumbilical incision. Four trocars were placed in the upper abdomen (Figure 1A). Perigastric lymph nodes were dissected through the main operating hole of abdominal cavity (Figure 1B). A trocar would be put into the thoracic cavity as the auxiliary port of LMLD (Figure 1C).

\section{Perigastric lymph node dissection}

The liver was retracted to expose the esophageal hiatus before lymphadenectomy. Total or proximal gastrectomy was performed based on the tumor staging and the clinician's judgment. Perigastric lymphadenectomy was performed in the order of No.4sb, $4 \mathrm{sa} \rightarrow$ No.11d, $2 \rightarrow$ No.4d, $6 \rightarrow$ No.5, 12a $\rightarrow$ No.7, 8a, 9,11p $\rightarrow$ No.1, 3 during total gastrectomy and in the order of No.4sb, 4sa $\rightarrow$ No.11d, $2 \rightarrow$ No.7, 8a, 9,11p $\rightarrow$ No.12a $\rightarrow$ No.1, 3 during proximal gastrectomy. The signal of the completion 

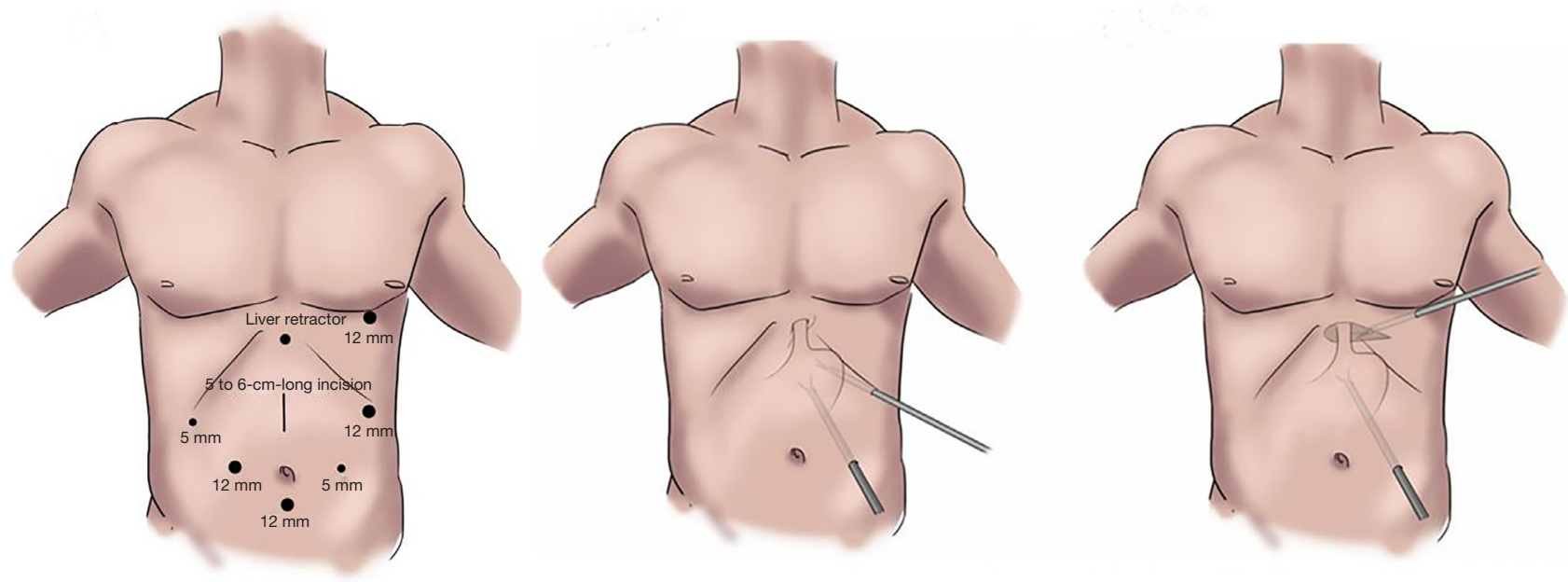

Figure 1 Distribution of trocars.
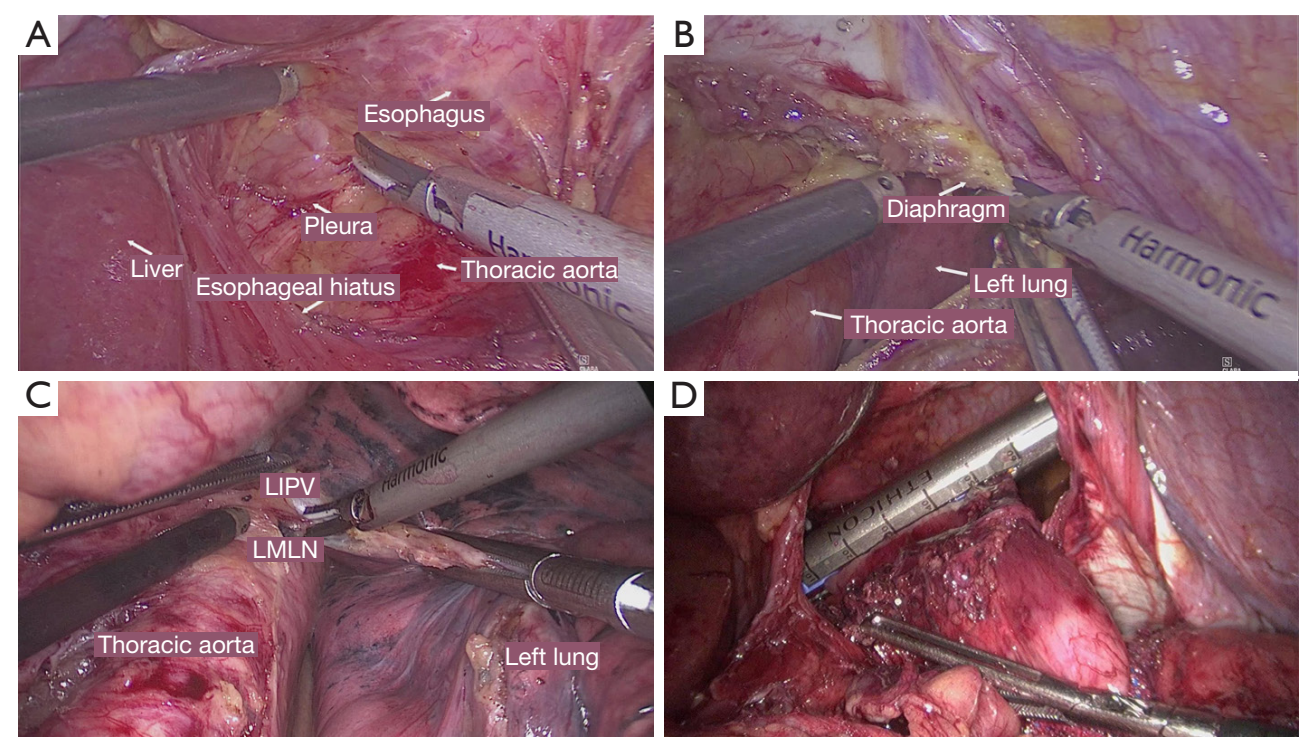

Figure 2 Lower mediastinal lymph node dissection. (A) Exposure of the infracardiac bursa; (B) opening the left diaphragm; (C) lower mediastinal lymphadenectomy; (D) transection of the esophagus. LMLN, lower mediastinal lymph nodes; LIPV, left inferior pulmonary vein.

of perigastric lymphadenectomy was the exposure of the abdominal esophagus and diaphragmatic crus.

\section{Paraesophageal laceration and LMLD}

After exposing the infracardiac bursa, the partial mediastinum tissue (No.112pulR, No.112aoA) was dissected (Figure $2 A$ ). After dividing the phrenicoesophageal ligament surrounding the esophagus, the No.19, 20 lymph nodes were removed. Then, the left diaphragm was incised $5-7 \mathrm{~cm}$ and the left thoracic cavity was connected with the mediastinum, which expanded the surgical field (Figure 2B). Subsequently, a $12 \mathrm{~mm}$ trocar was inserted into the 6-7th intercostal space of the left anterior axillary line as the main operation hole of the thoracic cavity. The upper boundary of LMLD was the left inferior pulmonary vein, the lower boundary was the esophageal hiatus, the anterior boundary was the pericardium, the posterior boundary was the thoracic 
Table 1 Preoperative data

\begin{tabular}{lc}
\hline Variables & Number $(\mathrm{n}=35)$ \\
\hline Sex (male/female) & $30 / 5$ \\
Age (years) & $67.0(50.0-83.0)$ \\
BMI $\left(\mathrm{kg} / \mathrm{m}^{2}\right)$ & $22.5(15.2-27.7)$ \\
Neoadjuvant therapy $(+/-)$ & $2 / 33$ \\
ASA score (I/II/III) & $0 / 22 / 13$ \\
\hline
\end{tabular}

BMI, body mass index; ASA, American Society of Anesthesiology.

Table 2 Operative and pathological data

\begin{tabular}{|c|c|}
\hline Variables & Statistical value \\
\hline \multicolumn{2}{|l|}{ Operative time (min) } \\
\hline Proximal (8 cases) & $247.5(195.0-275.0)$ \\
\hline Total gastrectomy ( 27 cases) & $290.0(173.0-530.0)$ \\
\hline Lower mediastinal LN dissection time (min) & $41.5(20.0-57.0)$ \\
\hline Blood loss (mL) & $100.0(20.0-200.0)$ \\
\hline Length of diaphragm incision (cm) & $6[5-7]$ \\
\hline \multicolumn{2}{|l|}{ No. of harvested LNs } \\
\hline Proximal (8 cases) & 26 [21-43] \\
\hline Total gastrectomy (27 cases) & 37 [21-63] \\
\hline \multicolumn{2}{|l|}{ No. of abdominal harvested LNs } \\
\hline Proximal (8 cases) & $21[16-32]$ \\
\hline Total gastrectomy (27 cases) & 32 [16-52] \\
\hline No. of harvested mediastinal LNs & 5 [2-13] \\
\hline Rate of metastatic mediastinal LNs ( $n, \%)$ & $9(25.7)$ \\
\hline \multicolumn{2}{|l|}{ pT stage $(n, \%)$} \\
\hline $\mathrm{T} 1$ & $1(2.9)$ \\
\hline T2 & $2(5.7)$ \\
\hline T3 & $14(40.0)$ \\
\hline $\mathrm{T} 4$ & $18(51.4)$ \\
\hline \multicolumn{2}{|l|}{ pN stage $(n, \%)$} \\
\hline No & $11(31.4)$ \\
\hline $\mathrm{N} 1$ & $5(14.3)$ \\
\hline N2 & $8(22.9)$ \\
\hline N3 & $11(31.4)$ \\
\hline
\end{tabular}

LN, lymph node. aorta, and the bilateral boundary was the bilateral pleura. The supradiaphragmatic nodes (No. 111) were dissected primarily from the abdominal operation hole, while the lower thoracic paraesophageal nodes (No. 110) and the posterior mediastinal nodes (No.112aoA, 112pul) were dissected from the thoracic operation hole (Figure 2C). The esophagus was transected $5 \mathrm{~cm}$ above the tumor (Figure 2D).

\section{Reconstruction}

Digestive tract reconstruction was performed after the negative margin was confirmed by intraoperative rapid frozen section. We put a drainage tube in the chest routinely and closed the diaphragm incision using an absorbable suture to prevent a hiatus hernia.

\section{Statistical analysis}

The data were analyzed using SPSS 23.0. Enumeration data were expressed as numbers and percentages, whilst the measurement data were expressed as medians (min-max).

\section{Results}

From May 2017 to December 2020, transthoracic single-port assisted laparoscopic esophagogastrectomy was performed in 35 patients ( 30 males and 5 females) with Siewert type II AEG. The median age was 67.0 (50.0-83.0) years old, and the median BMI was $22.5(15.2-27.7) \mathrm{kg} / \mathrm{m}^{2}$. Two patients received neoadjuvant therapy. Detailed clinical characteristics of the participants are shown in Table 1.

Table 2 shows the surgical and pathological results. There were no conversions to open surgery. Eight patients underwent proximal gastrectomy while the other 27 patients underwent total gastrectomy. The median operative times were 247.5 (195.0-275.0) min for proximal and $290.0(173.0-530.0) \mathrm{min}$ for total gastrectomy. The median time of LMLD was 41.5 (20.0-57.0) $\mathrm{min}$ and the median estimated blood loss was $100.0(20.0-200.0) \mathrm{mL}$. As for lymphadenectomy, the median numbers of total harvested lymph nodes in proximal and total gastrectomy were 26 [21-43] and 37 [21-63], respectively. The median number of harvested mediastinal lymph nodes was 5 [2-13]. Lower mediastinal lymph node metastasis occurred in 9 patients (25.7\%). There were 9 patients with esophageal 
Table 3 Metastasis rates in lower mediastinal lymph nodes

\begin{tabular}{|c|c|c|c|}
\hline \multirow{2}{*}{ Variables } & \multirow{2}{*}{ Lower mediastinal lymph nodes metastasis } & \multicolumn{2}{|c|}{ Length of esophageal involvement } \\
\hline & & $>2 \mathrm{~cm}$ & $\leq 2 \mathrm{~cm}$ \\
\hline \multirow{3}{*}{$\begin{array}{l}\text { Lower mediastinal lymph nodes } \\
\text { metastasis }(n, \%)\end{array}$} & Yes & $5(14.3)$ & $4(11.4)$ \\
\hline & No & $4(11.4)$ & $22(62.9)$ \\
\hline & $P$ & \multicolumn{2}{|c|}{0.030} \\
\hline
\end{tabular}

Table 4 Postoperative data

\begin{tabular}{lc}
\hline Variables & Number ( $\mathrm{n}=35)$ \\
\hline Mortality (+/-) & $0 / 35$ \\
Postoperative complication (+/-) & $4 / 31$ \\
Anastomotic leakage (+/-) & $1 / 34$ \\
Pleural effusion (+/-) & $2 / 33$ \\
Pancreatic fistula (+/-) & $1 / 34$ \\
Reoperation (+/-) & $1 / 34$ \\
Time to flatus (days) & $3[2-6]$ \\
Time to oral intake (days) & $6[2-14]$ \\
Extraction time of thoracic drainage tube (days) & $5[3-51]$ \\
Postoperative hospital stay (days) & $10[6-73]$ \\
\hline
\end{tabular}

involvement exceeding $2 \mathrm{~cm}$. Among them, 5 (55.6\%) had lower mediastinal lymph node metastasis. In patients with esophageal involvement no more than $2 \mathrm{~cm}$, the lower mediastinal lymph node metastasis rate was $15.4 \%$ (4/26). The lower mediastinal lymph node metastasis rate was significantly higher in patients with esophageal involvement exceeding $2 \mathrm{~cm}(\mathrm{P}=0.03)$. The data of lower mediastinal lymph node metastasis is shown in Table 3.

Postoperative complications were classified according to the Clavien-Dindo classification (11). Four patients suffered postoperative complications. One patient underwent reoperation due to an abdominal infection and anastomotic leakage, which were defined as Clavien-Dindo IIIb complications. Two patients suffered grade II events, including 1 case of pleural effusion and 1 case of pancreatic fistula. Another 1 patient underwent thoracocentesis due to pleural effusion, which was defined as a Clavien-Dindo IIIa complication. The median postoperative hospital stay was 10 [6-73] days. The median times to flatus and oral intake were 3 [2-6] and 6 [2-14] days, respectively. Detailed postoperative data are shown in Table 4.

\section{Discussion}

Laparoscopic distal gastrectomy can be considered as an alternative to treat early gastric cancer and advanced gastric cancer $(6,12)$. There is no prospective trial reported on laparoscopic total gastrectomy and laparoscopic esophagogastrectomy at present. With the development of laparoscopic technology and the popularization of the minimally invasive concept, laparoscopic techniques have been gradually used for advanced Siewert type II AEG $(8,9,13)$. Compared with traditional open transabdominal and the left thoracoabdominal (LTA) approach surgery, laparoscopic surgery has obvious advantages of minimal invasiveness. However, a standard procedure for Siewert type II AEG has not yet been established. Based on the experience of esophagogastrectomy through the LTA, our center has explored the application of the transthoracic single-port assisted laparoscopic approach for the treatment of advanced Siewert type II AEG, which can reduce the difficulty of LMLD and digestive tract reconstruction by the incision of the diaphragm and enhancement of the thoracic auxiliary port.

The survival benefit of LMLD has been a debatable issue for Siewert II AEG (14,15). Rüdiger Siewert et al. (16) found that the metastasis rate of lower mediastinal lymph nodes was $15.6 \%$ after a retrospective analysis of 1,002 consecutive AEG patients. Another multicenter retrospective study reported that the lower mediastinal lymph node metastasis rate was $17.7 \%$ in Siewert type II AEG $(17,18)$. The metastasis rate of lower mediastinal lymph nodes was related to the length of esophageal invasion and the $T$ stage of the tumor (17). Lower mediastinal lymph node metastasis predicts poor outcomes (19). According to the 13th edition of the Japanese Classification of Gastric Carcinoma, when the lymph node metastasis rate is $>10 \%$, dissection is strongly recommended. On the contrary, if the lymph node metastasis rate is $<5 \%$, it is not recommended (20). In our study, lower mediastinal lymph node metastasis occurred in $9(25.7 \%)$ patients who had a pT3-4 tumor. The metastasis 
rate in patients with esophageal invasion exceeding $2 \mathrm{~cm}$ was significantly higher than in patients with esophageal invasion no more than $2 \mathrm{~cm}(\mathrm{P}=0.030)$. Therefore, complete LMLD is recommended.

For the surgical approach of AEG, the right thoracoabdominal approach has more advantages in dissecting mediastinal lymph nodes, ensuring negative esophageal margin and gastrointestinal reconstruction. However, it needs to change the position during the operation $(21,22)$. The Dutch trial demonstrated that there was no significant overall survival benefit for extended transthoracic resection or transhiatal resection for Siewert type I and II AEG (23). The JCOG9502 trial revealed that the LTA approach conferred no survival benefit but increased the in-hospital mortality and postoperative morbidity for Siewert type II AEG compared with the TH approach. Hence, the TH approach was recommended to patients with Siewert type II AEG when the esophageal invasion was within $3 \mathrm{~cm}$ (5). These 2 randomized trials have provided a good evidence-based medical foundation for the selection of open surgical approaches for Siewert type II AEG. Currently, the laparoscopic TH approach has been widely used in Siewert type II AEG (24). Due to the obstruction of the diaphragm and surrounding tissue, LMLD, high esophageal denudation, and reconstruction of the digestive tract can be challenging. The open left diaphragm approach was developed to overcome these problems (25). However, owing to the deep position of the mediastinum and the obvious obstruction of the heart and thoracic aorta, it is still difficult to perform the operation by laparoscopy alone. Therefore, we inserted a $12 \mathrm{~mm}$ trocar where the thoracic drainage tube would be put to address these problems.

For digestive tract reconstruction, the commonly used reconstruction methods include esophagogastrostomy, roux-en-Y reconstruction, double tract reconstruction and so on. Esophagogastrostomy is the simplest and most widely used one. But severe reflux esophagitis may occur after operation, leading to poor postoperative quality of life (26). Roux-en-Y reconstruction and double tract reconstruction can effectively reduce the incidence of postoperative reflux esophagitis. Compare with roux-en-Y reconstruction, double tract reconstruction takes longer time in reconstruction, but the antireflux effect is better. There was no significant difference in long-term survival $(27,28)$. The way of digestive tract reconstruction can be selected according to the situation of patients. As for esophagogastrostomy and esophagojejunostomy, Overlap method, which is safer more feasible with a low incidence of complications (29), is commonly used in our center. The advantages of our technique are that the incision of the left diaphragm expanded the operation space, and the additional thoracic auxiliary port reduced interference between hands of operator.

The postoperative morbidity rate was $11.4 \%$ in our study, including 1 case of pancreatic fistula, 1 case of anastomotic leakage, and 2 cases of pleural effusion, lower than reported previously $(18,30)$. No patient used a ventilator after the operation. This meant than even if the diaphragm was incised to enter the thoracic cavity, there was no increase in thoracic complications. However, some experts doubt that overlong diaphragm incision will affect diaphragm movement and the lung function of patients. We are in the process of conducting relevant postoperative examinations to clarify this problem. In some cases, upper gastrointestinal angiography was performed after the operation, and it was found that the movement of the bilateral diaphragm was balanced, which had little effect on the respiratory movement.

In conclusion, transthoracic single-port assisted laparoscopic esophagogastrectomy for Siewert type II AEG is safe and feasible. However, the number of cases in this study is small. The long-term survival prognosis of patients is not yet clear, and further large-scale research is needed to confirm this.

\section{Acknowledgments}

Funding: Project of "Double First Class" and High-level University Discipline Collaborative Innovation Team of Guangzhou University of Chinese Medicine (2021xk48).

\section{Footnote}

Reporting Checklist: The authors have completed the STROBE reporting checklist. Available at https://dx.doi. org/10.21037/atm-21-4574

Data Sharing Statement: Available at https://dx.doi. org/10.21037/atm-21-4574

Conflicts of Interest: All authors have completed the ICMJE uniform disclosure form (available at https://dx.doi. org/10.21037/atm-21-4574). The authors have no conflicts of interest to declare.

Ethical Statement: The authors are accountable for all 
aspects of the work in ensuring that questions related to the accuracy or integrity of any part of the work are appropriately investigated and resolved. All procedures performed in this study involving human participants were in accordance with the Declaration of Helsinki (as revised in 2013). The study was approved by the ethics committee of Guangdong Provincial Hospital of Chinese Medicine (ZF2018-219) and informed consent was taken from all the patients.

Open Access Statement: This is an Open Access article distributed in accordance with the Creative Commons Attribution-NonCommercial-NoDerivs 4.0 International License (CC BY-NC-ND 4.0), which permits the noncommercial replication and distribution of the article with the strict proviso that no changes or edits are made and the original work is properly cited (including links to both the formal publication through the relevant DOI and the license). See: https://creativecommons.org/licenses/by-nc-nd/4.0/.

\section{References}

1. Mullen JT, Kwak EL, Hong TS. What's the Best Way to Treat GE Junction Tumors? Approach Like Gastric Cancer. Ann Surg Oncol 2016;23:3780-5.

2. Siewert JR, Hölscher AH, Becker K, et al. Cardia cancer: attempt at a therapeutically relevant classification. Chirurg 1987;58:25-32.

3. Japanese Gastric Cancer Association. Japanese gastric cancer treatment guidelines 2018 (5th edition). Gastric Cancer 2021;24:1-21.

4. Sasako M, Sano T, Yamamoto S, et al. Left thoracoabdominal approach versus abdominal-transhiatal approach for gastric cancer of the cardia or subcardia: a randomised controlled trial. Lancet Oncol 2006;7:644-51.

5. Kurokawa Y, Sasako M, Sano T, et al. Ten-year followup results of a randomized clinical trial comparing left thoracoabdominal and abdominal transhiatal approaches to total gastrectomy for adenocarcinoma of the oesophagogastric junction or gastric cardia. Br J Surg 2015;102:341-8.

6. Yu J, Huang C, Sun Y, et al. Effect of Laparoscopic vs Open Distal Gastrectomy on 3-Year Disease-Free Survival in Patients With Locally Advanced Gastric Cancer: The CLASS-01 Randomized Clinical Trial. JAMA 2019;321:1983-92.

7. Hyung WJ, Yang HK, Park YK, et al. Long-Term Outcomes of Laparoscopic Distal Gastrectomy for
Locally Advanced Gastric Cancer: The KLASS-02-RCT

Randomized Clinical Trial. J Clin Oncol 2020;38:3304-13.

8. Huang CM, Lv CB, Lin JX, et al. Laparoscopic-assisted versus open total gastrectomy for Siewert type II and III esophagogastric junction carcinoma: a propensity scorematched case-control study. Surg Endosc 2017;31:3495-503.

9. Sugita S, Kinoshita T, Kaito A, et al. Short-term outcomes after laparoscopic versus open transhiatal resection of Siewert type II adenocarcinoma of the esophagogastric junction. Surg Endosc 2018;32:383-90.

10. Ma F, Wang W, Guo D, et al. Short-term outcomes of laparoscopic versus open proximal gastrectomy with double-tract reconstruction for Siewert type II and III adenocarcinoma of the esophagogastric junction: a retrospective observational study of consecutive patients. Ann Transl Med 2021;9:352.

11. Dindo D, Demartines N, Clavien PA. Classification of surgical complications: a new proposal with evaluation in a cohort of 6336 patients and results of a survey. Ann Surg 2004;240:205-13.

12. Kim HH, Han SU, Kim MC, et al. Effect of Laparoscopic Distal Gastrectomy vs Open Distal Gastrectomy on Long-term Survival Among Patients With Stage I Gastric Cancer: The KLASS-01 Randomized Clinical Trial. JAMA Oncol 2019;5:506-13.

13. Kinoshita T, Gotohda N, Kato Y, et al. Laparoscopic transhiatal resection for Siewert type II adenocarcinoma of the esophagogastric junction: operative technique and initial results. Surg Laparosc Endosc Percutan Tech 2012;22:e199-203.

14. Lee IS, Ahn JY, Yook JH, et al. Mediastinal lymph node dissection and distal esophagectomy is not essential in early esophagogastric junction adenocarcinoma. World J Surg Oncol 2017;15:28.

15. Kurokawa Y, Hiki N, Yoshikawa T, et al. Mediastinal lymph node metastasis and recurrence in adenocarcinoma of the esophagogastric junction. Surgery 2015;157:551-5.

16. Rüdiger Siewert J, Feith M, Werner M, et al. Adenocarcinoma of the esophagogastric junction: results of surgical therapy based on anatomical/topographic classification in 1,002 consecutive patients. Ann Surg 2000;232:353-61.

17. Matsuda T, Kurokawa Y, Yoshikawa T, et al. Clinicopathological Characteristics and Prognostic Factors of Patients with Siewert Type II Esophagogastric Junction Carcinoma: A Retrospective Multicenter Study. World J Surg 2016;40:1672-9. 
18. Kurokawa Y, Takeuchi H, Doki Y, et al. Mapping of Lymph Node Metastasis From Esophagogastric Junction Tumors: A Prospective Nationwide Multicenter Study. Ann Surg 2021;274:120-7.

19. Hosokawa Y, Kinoshita T, Konishi M, et al. Clinicopathological features and prognostic factors of adenocarcinoma of the esophagogastric junction according to Siewert classification: experiences at a single institution in Japan. Ann Surg Oncol 2012;19:677-83.

20. Japanese Gastric Cancer Association. Japanese classification of gastric carcinoma--2nd English edition-response assessment of chemotherapy and radiotherapy for gastric carcinoma: clinical criteria. Gastric Cancer 2001;4:1-8.

21. Blank S, Schmidt T, Heger P, et al. Surgical strategies in true adenocarcinoma of the esophagogastric junction (AEG II): thoracoabdominal or abdominal approach? Gastric Cancer 2018;21:303-14.

22. Ott K, Bader FG, Lordick F, et al. Surgical factors influence the outcome after Ivor-Lewis esophagectomy with intrathoracic anastomosis for adenocarcinoma of the esophagogastric junction: a consecutive series of 240 patients at an experienced center. Ann Surg Oncol 2009;16:1017-25.

23. Omloo JM, Lagarde SM, Hulscher JB, et al. Extended transthoracic resection compared with limited transhiatal resection for adenocarcinoma of the mid/distal esophagus: five-year survival of a randomized clinical trial. Ann Surg 2007;246:992-1000; discussion 1000-1.

24. Sugita S, Kinoshita T, Kuwata T, et al. Long-term oncological outcomes of laparoscopic versus open transhiatal resection for patients with Siewert type II

Cite this article as: Luo $\mathrm{S}, \mathrm{Xu} \mathrm{J}$, Xiong W, Li J, Luo L, Zheng Y, Zeng H, Liu Y, Yang L, Wu Z, Yang X, Wang W. Feasibility and efficacy of transthoracic single-port assisted laparoscopic esophagogastrectomy for Siewert type II adenocarcinoma of the esophagogastric junction. Ann Transl Med 2021;9(20):1540. doi: 10.21037/atm-21-4574 adenocarcinoma of the esophagogastric junction. Surg Endosc 2021;35:340-8.

25. Takiguchi S, Miyazaki Y, Shinno N, et al. Laparoscopic mediastinal dissection via an open left diaphragm approach for advanced Siewert type II adenocarcinoma. Surg Today 2016;46:129-34.

26. Tokunaga M, Ohyama S, Hiki N, et al. Endoscopic evaluation of reflux esophagitis after proximal gastrectomy: comparison between esophagogastric anastomosis and jejunal interposition. World J Surg 2008;32:1473-7.

27. Li Z, Dong J, Huang Q, et al. Comparison of three digestive tract reconstruction methods for the treatment of Siewert II and III adenocarcinoma of esophagogastric junction: a prospective, randomized controlled study. World J Surg Oncol 2019;17:209.

28. Xiao JW, Liu ZL, Ye PC, et al. Clinical comparison of antrum-preserving double tract reconstruction vs rouxen-Y reconstruction after gastrectomy for Siewert types II and III adenocarcinoma of the esophagogastric junction. World J Gastroenterol 2015;21:9999-10007.

29. Ko CS, Gong CS, Kim BS, et al. Overlap method versus functional method for esophagojejunal reconstruction using totally laparoscopic total gastrectomy. Surg Endosc 2021;35:130-8.

30. Straatman J, van der Wielen N, Nieuwenhuijzen GA, et al. Techniques and short-term outcomes for total minimally invasive Ivor Lewis esophageal resection in distal esophageal and gastroesophageal junction cancers: pooled data from six European centers. Surg Endosc 2017;31:119-26.

(English Language Editor: C. Betlazar-Maseh) 